\title{
Dissipative Dynamics in a Quantum Register
}

\author{
P. Zanardii ${ }^{1,2}$ \\ 1 ISI Foundation, Villa Gualino \\ Viale Settimio Severo 65 -101133 Torino, Italy \\ 2 Unità INFM, Politecnico di Torino, \\ Corso Duca degli Abruzzi 24, I-10129 Torino, Italy
}

\begin{abstract}
A model for a quantum register dissipatively coupled with a bosonic thermal bath is studied The register consists of $N$ qubits (i.e. spin $\frac{1}{2}$ degrees of freedom), the bath is described by $N_{b}$ bosonic modes. The register-bath coupling is chosen in such a way that the total number of excitations is conserved. The Hilbert space splits allowing the study of the dynamics separately in each sector. Assuming that the coupling with the bath is the same for all qubits, the excitation sectors have a further decomposition according the irreducible representations of the $s u(2)$ spin algebra. The stability against environment-generated noise of the information encoded in a quantum state of the register depends on its $s u(2)$ symmetry content. At zero temperature we find that states belonging to the vacuum symmetry sector have for long time vanishing fidelity, whereas each lowest spin vector is decoupled from the bath and therefore is decoherence free. Numerical results are shown in the one-excitation space in the case qubit-dependent bath-system coupling.
\end{abstract}

71.10.Ad, 05.30.Fk

\section{INTRODUCTION}

The unavoidable interaction that each real-world system has with its environment is one of the major limitations to pratical realization of a quantum computer [1]. Indeed the outstanding potential capabilities of such a device rely heavily on the possibility of maintaining the quantum coherence in the system, and one of the typical effects of the coupling with the environment is to destroy phase relations between quantum states appearing in a linear superposition. This latter phenomenon is referred to as decoherence [2]: it can take place also when there is no system-environment energy exchange at all. To overcome this difficulty, in the last few years there has been a growing interest in the so-called error correction schemes [3], in which, by means of suitable encondings and measurement protocols, one is able to disentangle the system from the environment in order to recover uncorrupted information. Another possible approach, pointed out in [ [ is to make use of symmetry to protect the information stored in a quantum state against environment-induced noise. In this case one, rather than to design states that can be easily corrected, looks for states that cannot easily be disturbed. The simplest system for which dynamical symmetry provides us these "safe" states is a collection of $N$ qubits coupled all in the same way with the environment. Such a system can be thought of as quantum register $S$, analogous to those characteristic of classical computation. In this paper we study a model Hamiltonian describing the exchange of elementary quanta between the register and the environment, modelled by a bosonic bath $B$. The coupling with the bath is realized in term of the off-diagonal generators of a $s u(2)$ dynamical algebra [5]. The marginal dynamics of $S$ is dissipative (i.e. the register energy is not conserved). The global Hilbert space decomposes in (dynamically) invariant sec- tors, characterized by their $s u(2)$ symmetry content as well as their number of excitations, as it will be specified later. It should be emphasized that the focus of this paper is on the role played by dynamical-algebraic structures in providing collective states of the register intrinsecally stable against environment-induced decoherence. The adopted physical model is, in a sense, generic and it is not aimed to describe a specific physical implementation of a Quantum Computer (as done instead, for example in [6]) but a broad class of open quantum systems that could eventually turn out to be relevant for quantum data processing applications.

In sect. II, after recalling the fundamentals of open quantum systems, the model is introduced and its general feature briefly discussed. In sect. III the associated Hilbert space structure is analyzed. In sect. IV the oneexcitation subspace is studied, analytical as well as numerical results are presented for the fidelity and entropy. Sect. V contains some preliminary numerical results in the case of a qubit depending coupling with the bath. Section VI contains a number of conclusive remarks and perspectives

\section{THE MODEL}

Before introducing our model we begin by briefly recalling a few basic facts about open quantum systems. Let $\mathcal{H}_{s}, \mathcal{H}_{b}$ denote respectively the system and the environment Hilbert spaces. We assume $\mathcal{H}_{b}$ to be much larger than $\mathcal{H}_{s}$. The total Hilbert space is given by the tensor product $\mathcal{H}=\mathcal{H}_{s} \otimes \mathcal{H}_{b}$. A state over $\mathcal{H}_{\alpha}(\alpha=s, b)$ is a hermitean non negative operator $\rho_{\alpha}$ of $\operatorname{End}\left(\mathcal{H}_{\alpha}\right)$ with $\operatorname{tr}^{\alpha}\left(\rho_{\alpha}\right)=1$. The manifold of the state over $\mathcal{H}_{\alpha}$ will be denoted by $\mathcal{S}_{\alpha}$. The elements of $\mathcal{S}_{\alpha}$ that are also projectors $\left(\rho^{2}=\rho\right)$ provide the pure states. The set $\mathcal{S}_{\alpha}^{P}$ of 
pure states generates $\mathcal{S}_{\alpha}$ as its convex hull, furthermore it is in a one-to-one correspondence with $\mathcal{H}_{\alpha}$. According to quantum mechanics, time evolution of the overall (closed) system is unitary, therefore if the initial state has the separable form $\rho(0)=\rho_{s} \otimes \rho_{b},\left(\rho_{\alpha} \in \mathcal{S}_{\alpha}\right)$ then for any $t \geq 0$, the marginal (Liouvillian) evolution on $\mathcal{S}_{s}$ (open) is given by

$$
\mathcal{E}_{t}^{\rho_{b}}: \mathcal{S}_{s} \rightarrow \mathcal{S}_{s}: \rho_{s} \rightarrow \operatorname{tr}^{b}\left(U_{t} \rho(0) U_{t}^{\dagger}\right)
$$

where $\operatorname{tr}^{b}$ denotes the partial trace over $\mathcal{H}_{b}$. The superoperators $\left\{\mathcal{E}_{t}^{\rho_{b}}\right\}_{t \geq 0}$ are trace-preserving completely positive maps [7], that are the most general description of the evolution of an open quantum system. $\mathcal{S}_{\alpha}^{P}$ is not invariant under the action of $\left\{\mathcal{E}_{t}^{\rho_{b}}\right\}_{t \geq 0}$, typically an initial pure state of the subsystem becomes mixed in a very short time scale depending on the strength of the interaction. This state can either eventually get pure again or not, but in any case an irreversible loss of the information stored in the initial preparation has occurred. It is important to notice that this mechanism is active even when there is no energy-exchange between the subsystems (i.e. the subystem Hamiltonians are constants of motion) at finite as well as at zero temperature. When an energy-exchange occurs we call the resulting dynamics dissipative.

We introduce now the model. The Hamiltonian is given by $H=H_{s}+H_{b}+H_{I}$ where

$$
\begin{aligned}
& H_{s}=\sum_{i=1}^{N} \epsilon_{i} \sigma_{i}^{z}, \\
& H_{b}=\sum_{k=1}^{N_{b}} \omega_{k}\left(b_{k}^{\dagger} b_{k}+1 / 2\right), \\
& H_{I}=\sum_{i=1}^{N} \sum_{k=1}^{N_{b}}\left(g_{k i} b_{k}^{\dagger} \sigma_{i}^{-}+\text {h.c. }\right),
\end{aligned}
$$

here the $\sigma_{i}^{\alpha}$ 's are the spin 1/2 Pauli operators ( $i$ is the qubit index), and the $b_{k}$ 's bosonic operators. $H_{s}\left(H_{b}\right)$ is the Hamiltonian of the register (bath), $H_{I}$ the registerbath interaction. This model is closely related to the one known in the literature as the Dicke maser model [8]. The latter, for generic $N, N_{b}$, is not solvable and has a non-trivial ground-state phase diagram. In order to shed some light on the physics of this system we write down the equation of the motion for the Heisenberg operators $O(t) \equiv U(t)^{\dagger} O U(t)$. To simplify the expressions, it turns useful to perform the (unitary) transformation $\sigma_{j}^{ \pm} \mapsto \sigma_{j}^{ \pm} \exp \left( \pm i \epsilon_{i} t\right), b_{k} \mapsto b_{k} \exp \left(-i \omega_{k} t\right)$, whereby the Heisenberg equations then read

$$
\begin{aligned}
i \frac{\partial \sigma_{i}^{+}}{\partial t} & =2 \sum_{k} g_{k i}(t) b_{k}^{\dagger} \sigma_{i}^{z}, \\
i \frac{\partial \sigma_{i}^{z}}{\partial t} & =-\sum_{k}\left(g_{k i}(t) b_{k}^{\dagger} \sigma_{i}^{-}-\text {h.c. }\right) \\
i \frac{\partial b_{k}}{\partial t} & =-\sum_{j} g_{k j}(t) \sigma_{j}^{-} .
\end{aligned}
$$

where $g_{k i}(t) \equiv g_{k i} \exp \left[i\left(\omega_{k}-\epsilon_{i}\right) t\right]$. By a formal integration of the field equation and the substitution of the result into the spin equations one obtains

$$
\begin{aligned}
& i \frac{\partial \sigma_{i}^{+}}{\partial t}=\phi_{i}^{+}(t)-2 i \sum_{j} \int_{0}^{t} d \tau K_{i j}(t, \tau) \sigma_{j}^{+}(\tau) \sigma_{i}^{z}(t), \\
& i \frac{\partial \sigma_{i}^{z}}{\partial t}=-\phi_{i}^{z}(t)-\sum_{j} \int_{0}^{t} d \tau K_{i j}(t, \tau) \sigma_{j}^{+}(\tau) \sigma_{i}^{-}(t)-\text { h.c. }
\end{aligned}
$$

Here

$$
\begin{aligned}
K_{i j}\left(t, t^{\prime}\right) & =\sum_{k} g_{k i}(t) g_{k j}^{*}\left(t^{\prime}\right) \\
\phi_{i}^{+}(t) & =2 \sum_{k} g_{k i}(t) b_{k}^{\dagger}(0) \sigma_{i}^{z}(t), \\
\phi_{i}^{z}(t) & =\sum_{k} g_{k i}(t) b_{k}^{\dagger}(0) \sigma_{i}^{-}(t) .
\end{aligned}
$$

This coupled system of non-linear integro-differential equations describes the dynamics of the spin subsystem in closed form. By means of the intermediation of the bath bosons each spin gets interacting with all the others it via a sort of time-retarded Heisenberg coupling. The information about the bath (dynamics as well as preparation) is contained in the kernels $K_{i j}$, and in the operators $\left\{\phi_{i}^{\alpha}\right\}$. If $\epsilon_{j}=\epsilon,(j=1, \ldots, N)$ and the bathspin coupling is the same for all the spins, one has

$$
K_{i j}\left(t, t^{\prime}\right)=\sum_{k}\left|g_{k}\right|^{2} \exp \left[-i\left(\epsilon-\omega_{k}\right)\left(t-t^{\prime}\right)\right] \text {. }
$$

Under rather general assumptions this kernel is strongly peaked at $t=t^{\prime}$, If one has $K_{i j}\left(t, t^{\prime}\right) \sim \delta\left(t-t^{\prime}\right)$ then (6) become a system of coupled non-linear differential equations. Despite this strong simplification, also in this case the solution, due to non-linearity, remains difficult and one has to resort to numerical techniques.

An alternative approach based on symmetry considerations, will be introduced in the next section.

\section{HILBERT SPACE STRUCTURE}

The Hilbert space is given by the tensor product $\mathcal{H}=$ $\mathcal{H}_{s}^{\otimes N} \otimes \mathcal{H}_{b}^{\otimes N_{b}}$, being $\mathcal{H}_{s}\left(\mathcal{H}_{b}\right)$ the two (infinite) dimensional single spin (boson) space. The coupling of the spin system with the bosonic bath is described by the hamiltonian $H_{I}$ is such that the raising (lowering) of one spin state is associated to the destruction (creation) of one boson. From this follows that the system admits the constant of motion

$$
\mathcal{I}=\sum_{i=1}^{N} \sigma_{i}^{z}+\sum_{k=1}^{N_{b}} n_{k}+N / 2
$$


The eigenvalues of $\mathcal{I}$ give the number of elementary (spin as well as bosonic) excitations over the reference state $|0\rangle \equiv|0\rangle_{s} \otimes|0\rangle_{b}$. The latter is a lowest weight vector for the spin as well as for the boson algebra: $\sigma_{\alpha}^{-}|0\rangle=b_{k}|0\rangle=0, \forall \alpha, k$. Its energy is set equal to zero. The Hilbert space splits into invariant eigenspaces of $\mathcal{I}, \mathcal{H}=\oplus_{I} \mathcal{H}_{I}$; an elementary combinatorial argument shows that the dimension of the $I$-excitations space $\mathcal{H}_{I},(I \in \mathbf{N})$ is given by

$$
d_{I}=\sum_{l=0}^{\min (N, I)}\left(\begin{array}{c}
N \\
l
\end{array}\right)\left(\begin{array}{c}
I-l+N_{b}-1 \\
N_{b}-1
\end{array}\right)
$$

If $N=N_{b}=1$, one has $d_{0}=1, d_{I}=2,(I \geq 1)$, the model reduces to the exactly solvable Jaynes-Cummings model of quantum optics [9]. It is worth noticing that the general spin-boson model considered in the literature on quantum dissipation, [usually addressed in the framework of the Feynmann-Vernon influence functional (see 10 for a review)], due to the presence of terms $b_{k} \sigma_{i}^{-}, b_{k}^{\dagger} \sigma_{i}^{+}$, (neglected here in view of the rotating wave approximation) does not conserve $\mathcal{I}$, spoiling the associated dynamical decomposition of the Hilbert space, on which our subsequent numerical analysis relies. Nevertheless, since in this papers we are interested only in the role played by collective effects in stabilizing a quantum state, this restrictions does not result in any severe loss of generality.

A basis for $\mathcal{H}_{I}$ is given by $\left|\psi_{\alpha, k}^{(n)}\right\rangle=|\alpha\rangle \otimes|k\rangle$, where

$$
|\alpha\rangle \equiv \prod_{j=1}^{n} \sigma_{\alpha_{j}}^{+}|0\rangle_{s}, \quad|k\rangle \equiv \prod_{j=1}^{I-n} b_{k_{j}}^{\dagger}|0\rangle_{b}
$$

Where $n=1, \ldots, \min (I, N), \quad \alpha \in \mathcal{C}(N, n), \quad k \in$ $\mathcal{C}^{\prime}\left(N_{b}, N-n\right), \mathcal{C}(n, k)\left(\mathcal{C}^{\prime}(n, k)\right)$ denoting the set of the combinations without (with) repetitions of $n$ objects $k$ by $k$. Following the general scheme of [4] we specialize hereafter the model assuming the parameters $\left\{\epsilon_{i}\right\},\left\{g_{k i}\right\},(i=1, \ldots, N)$, independent of the qubit replica index $i$. The first assumptions follows simply from the fact that qubits are replicas of the same system. The independence of the coupling constants on the qubit index is analogous to the so-called Dicke limit of quantum optics [8]; it holds - for example - when the typical bath wave-lengths coupled with the register are much greater than the distances between the qubits. In this latter case the environment is no able to probe the internal structure of the register: as long as the dynamics is concerned it has an effective point-like topology. The common value $\epsilon$ of the qubit "magnetic" fields $\left\{\epsilon_{i}\right\}$ will be chosen as the unit of the energy scale; analogously the "sound velocity" of the boson will be set equal to one, so that their dispersion relation reads $\omega_{k}=k,\left(k=2 \pi n / N_{b}, n=1, \ldots, N_{b}\right)$. The Hamiltonian can then be written as

$$
H=\epsilon S^{z}+B S^{+}+S^{-} B^{\dagger}+H_{b},
$$

being $S^{\alpha}=\sum_{j=1}^{N} S_{j}^{\alpha},(\alpha=z, \pm)$ global spin operators, spanning a Lie Algebra $s u(2)$, and $B=\sum_{k} g_{k} b_{k}$.

The fact that in (10) the spins appear only trough the $S^{\alpha}$ 's means the all the qubits are treated symmetrically: the dynamics allows only for coherent excitations of the computational (spin) degrees of freedom. This is, from the algebraic point of view, a very strong constraint: the dynamics gets invariant under the action of the symmetryc group $\mathcal{S}_{N}$ of the qubit index permutations. This provides us one more constant of motion. Indeed from (10) immediately follows that the total spin $S^{2}$ is conserved and $\mathcal{H}_{I}$ splits according the $s u(2)$-irrep. The multiplicity of each irrep associated with the total spin quantum number $\mathrm{S}$ is given by

$$
n(S, N)=\frac{N !(2 S+1)}{(N / 2+S+1) !(N / 2-S) !} .
$$

One finds the following decomposition in invariant subspaces

$$
\begin{aligned}
\mathcal{H}_{I} & =\bigoplus_{S=S_{m}(N, I)}^{N / 2} \bigoplus_{r=1}^{n(S, N)} \mathcal{H}_{I}(S, r), \\
\mathcal{H}_{I}(S, r) & =\bigoplus_{S^{z}=-S}^{\min (I-N / 2, S)}\left|I, S, r, S^{z}\right\rangle \otimes \mathcal{H}_{b}\left(N_{b}\left(I, S^{z}\right)\right),
\end{aligned}
$$

where $S_{m}(N, I)=\max (N / 2-I, s),(s=0$ for $N$ even, and $s=1 / 2$ otherwise), $\mathcal{H}_{b}(N)$ denotes the eigenspace, in $\mathcal{H}_{b}$, of $N_{b}=\sum_{k} n_{k}$ corresponding to the eigenvalue $N, N_{b}\left(I, S^{z}\right)=I-N / 2-S^{z},\left|I, S, r, S^{z}\right\rangle$ is a simultaneous eigenvector of $\mathcal{I}, S^{2}, S^{z}$ associated respectively to the eigenvalues $I, S(S+1), S^{z}$. The reference state $|0\rangle$, belongs to the subspace $\oplus_{I} \mathcal{H}_{I}(N / 2)$, with maximal total spin eigenvalue $S=N / 2$ : this subspace will be denoted by $\mathcal{H}^{\text {sym }}$, and referred to as the symmetric subspace. If $\mathcal{H}_{I}^{\text {sym }} \equiv \mathcal{H}^{\text {sym }} \cap \mathcal{H}_{I}$, one has

$$
\begin{aligned}
\mathcal{H}_{I}^{\text {sym }} & =\operatorname{span}\left\{\left(S^{+}\right)^{n}\left|\psi_{0, k}^{(I-n)}\right\rangle \mid n=0, \ldots, I\right\} \\
\operatorname{dim}\left(\mathcal{H}_{I}^{s y m}\right) & =\sum_{l=0}^{\min (N, I)}\left(\begin{array}{c}
I-l+N_{b}-1 \\
N_{b}-1
\end{array}\right) .
\end{aligned}
$$

The orthogonal complement of $\mathcal{H}_{I}^{\text {sym }}$ will be denoted by $\mathcal{H}_{I}^{A}$. It is the direct sum of all the sectors with non-maximal $S^{2}$-eigenvalue. Before ending this section we notice that an additional term of the form $H^{\prime}=$ $S^{z} \sum_{k} w_{k}\left(b_{k}+b_{k}^{\dagger}\right)$, would destroy the $u(1)$ symmetry generated by $\mathcal{I}$, but not the $s u(2)$ structure. Such a term, considered in [4], does not correspond to an energyexchange but is a source of pure decoherence. Since our analysis relies on the invariant decomposition $\mathcal{H}=\oplus_{I} \mathcal{H}_{I}$ this term has been omitted here. 


\section{1-EXCITATION SPACE}

Due to the field-theoretic nature of our model, the dimensionality formula (8) clearly shows that for increasing excitation number $I$ the problem of diagonalizing $H$ becomes rapidly intractable. In particular, a finitetemperature analysis (arbitrary number of excitations) is very difficult. Nevertheless one of the interesting features of quantum noise is to be active also a $T=0$, thanks to vacuum fluctuations. This latter issue can be addressed by exact numerical means, without an artificious truncation of the bosonic space, by noticing that the one-excitation space $\mathcal{H}^{(1)}$, has dimension $d_{1}=N+N_{b}$, that is only a linear function of the total number of degrees of freedom. The basis $\left\{\left|\psi_{\alpha, k}^{(1)}\right\rangle\right\}$ is given by $|\alpha\rangle \equiv$ $\sigma_{\alpha}^{+}|0\rangle,(\alpha=1, \ldots, N)$ and $|k\rangle \equiv b_{k}^{\dagger}|0\rangle,\left(k=1, \ldots, N_{b}\right)$. Equation (13) in this case reads

$$
\mathcal{H}_{1}=\mathcal{H}_{1}(N / 2,1) \bigoplus_{r=1}^{N-1} \mathcal{H}_{1}(N / 2-1, r)
$$

The symmetric space $(S=N / 2)$ is $N_{b}+1$-dimensional and it is spanned by the vector $\left|\psi^{\text {sym }}\right\rangle \equiv N^{-1 / 2} S^{+}|0\rangle$, and by the whole set $\{|k\rangle\}$. The subspace $\mathcal{H}_{1}^{A}$ corresponds to $S=N / 2-1$. An orthonormal basis of $\mathcal{H}_{1}^{A}$ is given by

$$
\left|\phi_{k}\right\rangle \equiv S_{k}^{+}|0\rangle, \quad S_{k}^{+} \equiv N^{-1 / 2} \sum_{j=1}^{N} e^{i k j} \sigma_{j}^{+}
$$

where, $k=2 n \pi / N, n=1, \ldots, N-1$. Since in $\mathcal{H}_{1}^{A}$ the bosonic vacuum factorizes, this subspace, when necessary, will be identified with its projection over $\mathcal{H}_{s}$. Now we observe that the vectors $\left\{\left|\phi_{k}\right\rangle\right\}$, are annihilated by $S^{-}$, as they have minimal $S^{z}$-projection, but also by the $\left\{b_{k}\right\}$, as they have empty boson sector. From this follows that $H_{I}\left|\phi_{k}\right\rangle=0,(\forall k)$ therefore $\mathcal{H}_{1}^{A}$ is decoupled from the bath; it is an energy eigenspace with eigenvalue $E=\epsilon$. In terms of evolution superoperators, if $\rho$ is a state over $\mathcal{H}_{1}^{A}$, we have ithe fixed-point relations $\mathcal{E}_{t}^{0}(\rho)=\rho,(t \geq 0)$, where $\mathcal{E}_{t}^{0}$ denotes the superoperator associated with the bath-vacuum density matrix $|0\rangle_{b}\left\langle\left. 0\right|_{b}\right.$. The states over $\mathcal{H}_{1}^{A}$ are unaffected by the decoherence induced by coupling with the bath vacuum and can therefore to encode information in a safe way. The space $\mathcal{H}_{1}^{A}$ is noiseless only at zero temperature; for finite temperature the $\left|\phi_{k}\right\rangle$ 's get mixed with all the vectors belonging to the same $s u(2)$ irrep, making the induced dynamics non unitary. It is important to notice that, for $N \neq 2$, this states are not the noiseless ones introduced in [4], as the latter are associated with spin singlets (i.e. are annihilated by $S^{-}$ and $S^{+}$) and are decoherence-free at any temperature, whereas the $\left|\phi_{k}\right\rangle$ 's belong to $N$-1-dimensional su(2) multiplets. The spectrum in the symmetric subspace can be obtained by resorting to exact numerical diagonalization of $H$, that provides the eigenvectors and eigenvalues $\left\{\left|\phi_{i}\right\rangle, E_{i}\right\}_{i=1}^{d_{1}}$.
On the other hand the spectrum in $\mathcal{H}_{1}^{\text {sym }}$ is given by the $N_{b}+1$ zeros of the expression [1]

$$
P_{N, N_{b}}(E)=E-\epsilon-N \sum_{k=1}^{N_{b}} \frac{\left|g_{k}\right|^{2}}{E-\omega_{k}}
$$

that corresponds to the analogous single spin problem with rescaled coupling $g_{k} \mapsto \sqrt{N} g_{k}$. This follows from the symmetry constraint that makes $\left|\psi^{\text {sym }}\right\rangle$ the only state coupled with the bosonic modes. Let $\left|\psi_{0}\right\rangle=$ $\sum_{i} c_{i}^{0}\left|\phi_{i}\right\rangle,\left(c_{i}^{0}=\left\langle\phi_{i} \mid \psi_{0}\right\rangle\right)$ be the initial state; at $t>0$ we can write, in terms of the chosen basis

$$
\begin{aligned}
& |\psi(t)\rangle \equiv e^{-i H t}\left|\psi_{0}\right\rangle=\sum_{\gamma=1}^{d_{1}} C_{\gamma}(t)|\gamma\rangle \in \mathcal{H}, \\
& C_{\gamma}(t)=\sum_{i=1}^{d_{1}} c_{i}^{0} c_{\gamma}^{i} e^{-i E_{i} t}, \quad\left(c_{\gamma}^{i} \equiv\left\langle\gamma \mid \phi_{i}\right\rangle\right) .
\end{aligned}
$$

The marginal density matrix is given by $\rho_{s}(t)=$ $\operatorname{tr}^{b}|\psi(t)\rangle\langle\psi(t)|$. By using the relations

$$
\begin{aligned}
\operatorname{tr}^{b}|\alpha\rangle\left\langle\alpha^{\prime}\right| & =\sigma_{\alpha}^{+}|0\rangle_{s}\left\langle\left. 0\right|_{s} \sigma_{\alpha^{\prime}}^{-}, \operatorname{tr}^{b} \mid k\right\rangle\left\langle k^{\prime}\left|=\delta_{k k^{\prime}}\right| 0\right\rangle_{s}\left\langle\left. 0\right|_{s}\right. \\
\operatorname{tr}^{b}|\alpha\rangle\langle k| & =\operatorname{tr}^{b}|k\rangle\langle\alpha|=0
\end{aligned}
$$

one obtains

$$
\begin{aligned}
\rho_{s}(t) & =\sum_{\alpha \alpha^{\prime}=1}^{N} C_{\alpha}(t) \bar{C}_{\alpha^{\prime}}(t)|\alpha\rangle\left\langle\alpha^{\prime}\right| \\
& +|0\rangle_{s}\left\langle\left. 0\right|_{s} \sum_{k=1}^{N_{b}}\left|C_{k}(t)\right|^{2} .\right.
\end{aligned}
$$

The first (last) $N\left(N_{b}\right)$ terms describe a sector with a reversed (excited) spin (boson). The marginal density matrix can be readily diagonalized, simply by observing that it can be written in the form $\rho_{s}(t)=P_{1}(t)\left|\psi_{s}(t)\right\rangle\left\langle\psi_{s}(t)\right|+$ $P_{0}(t)|0\rangle_{s}\left\langle\left. 0\right|_{s}\right.$, where

$$
\left|\psi_{s}(t)\right\rangle=\frac{1}{\sqrt{P_{1}(t)}} \sum_{\alpha=1}^{N} C_{\alpha}(t)|\alpha\rangle \in \mathcal{H}_{s}^{\otimes N},
$$

and $P_{0}(t)=1-P_{1}(t)=\sum_{k=1}^{N_{b}}\left|C_{k}(t)\right|^{2}$. The von Neumann entropy of $S$ is therefore given by $S_{s}(t)=$ $-\sum_{i=0}^{1} P_{i}(t) \log _{2} P_{i}(t)$. A completely symmetric expression $\left(\alpha \leftrightarrow k, P_{1} \leftrightarrow P_{0}\right)$ is obtained for the bath marginal density matrix $\rho_{b}=\operatorname{tr}^{s}(\rho)$, from which follows that $S_{b}=S_{s}$, furthermore we have $S(S \mid B)=S(B \mid S)=-S_{s}$ for the conditional entropies, and $S(B: S)=-2 S_{s}$ for the mutual entropy; this is a consequence of the purity of the overall system-bath state.

In order to study the corruption of the information stored in a pure quantum state it appears useful to study the following quantity, called (input-output) fidelity 12]

$$
F(t)=\left\langle\psi_{0}^{s}\left|\rho_{s}(t)\right| \psi_{0}^{s}\right\rangle
$$


The fidelity measures the overlap between the initial state $\left|\psi_{0}^{s}\right\rangle$ and the evolved one. In the following we shall be interested in the evaluation of (21) with initial data in $\mathcal{H}_{1}$ of the form $\left|\psi_{0}\right\rangle=\left|\psi_{0}^{s}\right\rangle \otimes|0\rangle_{b},\left(\rho_{s}(0)=\left|\psi_{0}^{s}\right\rangle\left\langle\psi_{0}^{s}\right|\right)$. For such initial preparation, if $\left|\tilde{\psi}_{s}(t)\right\rangle=\sqrt{P_{1}(t)}\left|\psi_{s}(t)\right\rangle$, one can write

$$
\begin{aligned}
F(t) & =\left\langle\tilde{\psi}_{s}(t)\left|\rho_{s}(0)\right| \tilde{\psi}_{s}(t)\right\rangle \\
& =\left|\left\langle\psi_{0}^{s} \mid \tilde{\psi}_{s}(t)\right\rangle\right|^{2}=\left|\sum_{\alpha=1}^{N} C_{\alpha}(t) \bar{C}_{\alpha}(0)\right|^{2} \equiv|D(t)|^{2}
\end{aligned}
$$

The "decoherence" function $D(t)$ is related to the decay of the off-diagonal elements of $\rho_{s}(t)$; indeed if, at $t=0$, we prepare the system in the state $2^{-1 / 2}\left(|0\rangle_{s}+\right.$ $\left.\left|\psi_{0}^{s}\right\rangle\right) \otimes|0\rangle_{b}$, where $S^{z}\left|\psi_{0}^{s}\right\rangle=(1-N / 2)\left|\psi_{0}^{s}\right\rangle$, it is immediate to check that $\left\langle\psi_{0}^{s}\left|\rho_{s}(t)\right| 0\right\rangle_{s}=2^{-1} D(t)$. The mechanism responsible for the energy exchange induces also a dephasing between the zero and one excited spin states: dissipation is associated with decoherence.

In figure (11) is shown the behaviour of $F(t)$, for different values of the coupling with the bath, with initial data $\left|\psi_{0}^{s}\right\rangle=\left|\psi^{s y m}\right\rangle$. In this figure and in the subsequent ones $\epsilon^{-1}$ is chosen as the time unit. These results are obtained by exact diagonalization of $H$ in $\mathcal{H}_{1}$, that provides the dynamical functions $\left\{C_{\gamma}(t)\right\}$. For strong bath-system couplings $F(t)$ develops oscillatory structures, due to the back and forth exchange of energy between the system and the bath. We report the simulations for weak couplings, since it is the case physically relevant. Furthermore, since we are essentialy interested in the role played in the large times dynamics, by the symmetry structure of the initial state, and not in a detailed description of the bath-system coupling, we have choosen $g_{k}=g_{0},(\forall k)$. From the point of view of the energy-information loss the latter choice is the worst case in that each qubit is coupled equally well with all the bath modes, which should not, of course, be the case in real systems. The fidelity in this range of coupling parameters and for intermediate times, vanishes in exponential way $F(t) \simeq \exp (-t / \tau)$. The relaxation time $\tau$, which turns out to be inversely proportional to $N \sum_{k}\left|g_{k}\right|^{2}$, is the time scale over which the dissipative process takes place. The real and immaginary parts of $D(t)$ have an exponential damping modulated by oscillations over a time scale $\epsilon^{-1}$. For very small times a naive perturbation up to the second order in $H_{I}$ shows that indeed $F(t) \stackrel{t \rightarrow 0+}{\simeq} 1-t^{2} / 2 N \Delta$, where $\Delta=\sum_{k}\left|g_{k}\right|^{2}$. Of course this process is nothing but the relaxation of the excited spin, whose energy is transferred to the environment; for sufficiently large times one finds

$$
U_{t}\left|\psi^{s y m}\right\rangle \otimes|0\rangle_{b}=|0\rangle_{b} \otimes\left|\psi_{b}\right\rangle,
$$

where $\left|\psi_{b}\right\rangle=\sum_{k} c_{k}|k\rangle$, is a superposition of all one boson states.

Some words of caution are now in order. The model under consideration is nothing but a multi-mode generalization of the Jaynes-Cummings model with many atoms.
In analogy with the latter, for long time scale, $t>t_{C}$, it exhibits a complex pattern of collapses and revivals [13]. Furhermore since in each excitation space we have only a finite number of degrees of freeedom the phenomenon of the Poincaré recurrences is also present for $t>t_{R}$. In the following we will show results for $t \ll t_{C}, t_{R}$, in other terms we assume that, thanks to the great number of bosonic modes and the weak coupling, the physically relevant time-scales are much smaller than the ones at which this more complex behaviour appear. The energy exchange of the register with the bath can then be considered irreversible.

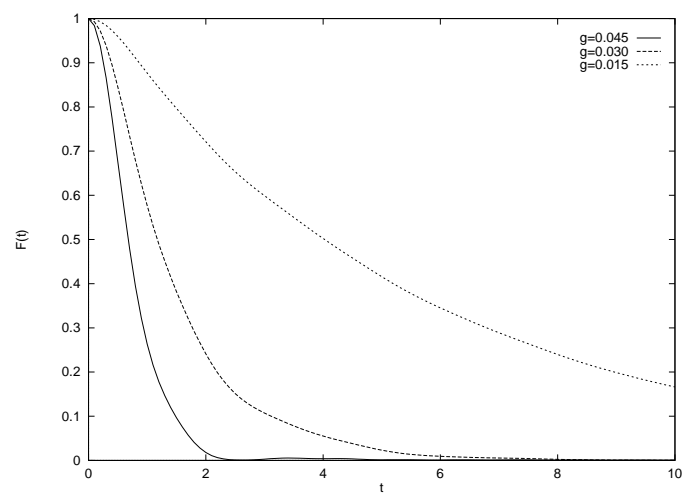

FIG. 1. Fidelity as a function of time for $\left|\psi_{0}\right\rangle=\left|\psi^{\text {sym }}\right\rangle$. The coupling function is $g_{k}(i)=g \forall k, i,\left(N=2, N_{b}=200\right)$. The time unit is $\epsilon^{-1}$.

Suppose now that $\left|\psi_{0}^{s}\right\rangle=c_{s}\left|\psi^{s y m}\right\rangle+c_{a}\left|\psi^{a}\right\rangle$, where $\left|\psi^{a}\right\rangle \in \mathcal{H}_{1}^{A}$, is a normalized vector, and $\left|c_{a}\right|^{2}+\left|c_{s}\right|^{2}=1$. Also, for the sake of concreteness, and without any loss of generality, let us consider the case $N=2$. The vectors $\left|\psi^{\text {sym }}\right\rangle,\left|\psi^{a}\right\rangle$, are given respectively by the two Bell-basis states $\left|\psi^{\text {sym }}\right\rangle=\frac{1}{\sqrt{2}}(|\uparrow \downarrow\rangle+|\downarrow \uparrow\rangle),\left|\psi^{a}\right\rangle=\frac{1}{\sqrt{2}}(|\uparrow \downarrow\rangle-|\downarrow \uparrow\rangle)$. The initial marginal density matrix of $S$ is therefore given by

$$
\begin{aligned}
\rho_{s}(0) & =\left|c_{s}\right|^{2}\left|\psi^{\text {sym }}\right\rangle\left\langle\left.\psi^{\text {sym }}|+| c_{a}\right|^{2} \mid \psi^{a}\right\rangle\left\langle\psi^{a}\right| \\
& +c_{s} \bar{c}_{a}\left|\psi^{s y m}\right\rangle\left\langle\psi^{a}\left|+\bar{c}_{s} c_{a}\right| \psi^{\alpha}\right\rangle\left\langle\psi^{s y m}\right|,
\end{aligned}
$$

By using our previous result for the symmetric initial state, and the fact that $\left|\psi^{a}\right\rangle$ is an energy eigenstate, it follows easily from (23), for $t$ large enough, that

$$
\begin{aligned}
\mathcal{E}_{t}^{0}\left(\left|\psi^{a}\right\rangle\left\langle\psi^{\text {sym }}\right|\right) & =\mathcal{E}_{t}^{0}\left(\left|\psi^{\text {sym }}\right\rangle\left\langle\psi^{a}\right|\right)=0 \\
\mathcal{E}_{t}^{0}\left(\left|\psi^{a}\right\rangle\left\langle\psi^{a}\right|\right) & =\left|\psi^{a}\right\rangle\left\langle\psi^{a}\left|, \mathcal{E}_{t}^{0}\left(\left|\psi^{\text {sym }}\right\rangle\left\langle\psi^{\text {sym }}\right|\right)=\right| 0\right\rangle_{s}\left\langle\left. 0\right|_{s}\right.
\end{aligned}
$$

Therefore the large times density matrix is given by

$$
\rho_{s} \simeq\left|c_{a}\right|^{2}\left|\psi^{a}\right\rangle\left\langle\left.\psi^{a}|+| c_{s}\right|^{2} \mid 0\right\rangle_{s}\left\langle\left. 0\right|_{s},\right.
$$

from which straightforwardly follows for the fidelity the behaviour 


$$
F \simeq\left|c_{a}\right|^{4}=\left(1-\left|c_{s}\right|^{2}\right)^{2}=\left(1-\left|\left\langle\psi^{s y m} \mid \psi_{0}^{s}\right\rangle\right|^{2}\right)^{2} .
$$

In other terms: the final state depends on the initial preparation symmetry content; a complete corruption of the initial information is obtained only if the initial state belongs to the vacuum $S^{2}$-sector $\mathcal{H}_{1}(N / 2)$, so that the smaller is the projection over it, the closer to one is the fidelity.

The extreme case is $\left|\psi_{0}^{s}\right\rangle \in \mathcal{H}_{1}^{A}, F(t)=1, \forall t$ in which there is no relaxation at all. In the intermediate situations the spin system remains partially entangled with the environment and its state never gets pure. This situation is illustrated in figures (2), and (3) where fidelity and entropy are shown as functions of time in the case of $\left|\psi_{0}^{s}\right\rangle=M^{-1 / 2} \sum_{\alpha=1}^{M}|\alpha\rangle$, for $M=1,2,3$. In this case it is trivial to check, by using equations (26), and (27), that $F \simeq(1-M / N)^{2}$, and $S_{s} \simeq(M / N-1) \log _{2}(1-M / N)-$ $M / N \log _{2}(M / N)$. Notice that if $\left|c_{s}\right|^{2}=1$, one has the complete de-excitation of the spin system, therefore the initial state $\left|\psi^{\text {sym }}\right\rangle$ is maximally entangled and the final state $|0\rangle_{s}$, with zero mutual entanglement of the qubits. The system undergoes energy as well as information loss. On the other hand if $\left|c_{a}\right|^{2}=1$, the final, and initial, state $\left|\psi_{a}\right\rangle$ is maximally entangled: energy and information are conserved.

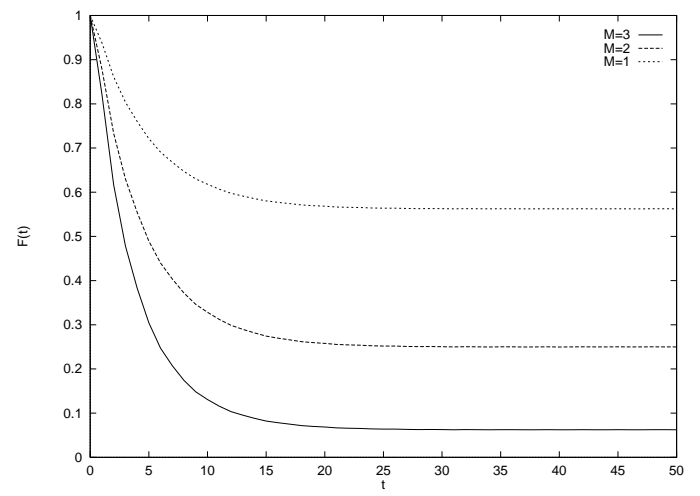

FIG. 2. Fidelity as a function of time for $\left|\psi_{0}^{s}\right\rangle=M^{-1 / 2} \sum_{\alpha=1}^{M}|\alpha\rangle,\left(M=1,2,3 ; N=4, N_{b}=200\right)$. $g_{k}(i)=0.01 \forall k, i$. The time unit is $\epsilon^{-1}$.

In the temporal range in which the decay of fidelity is exponential, the following relation between relaxation times holds: $\tau\left(c_{s}\right)\left|c_{s}\right|^{2}=\tau(1)$.

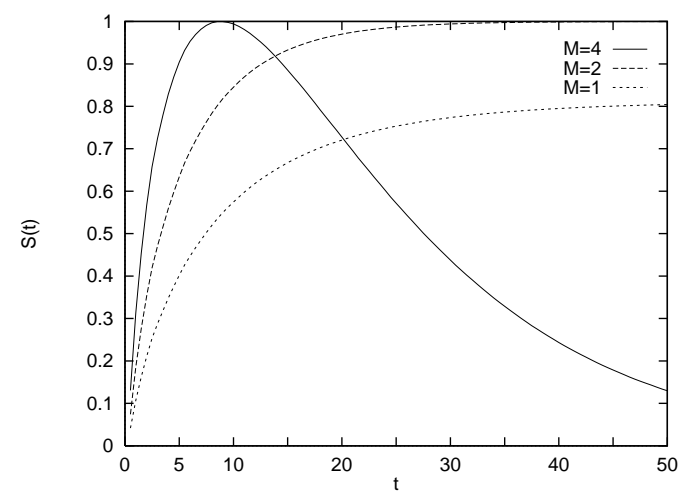

FIG. 3. Entropy as a function of time for $\left|\psi_{0}^{s}\right\rangle=M^{-1 / 2} \sum_{\alpha=1}^{M}|\alpha\rangle,\left(M=1,2,3 ; N=4, N_{b}=200\right)$. $g_{k}(i)=0.01 \forall k, i$. The time unit is $\epsilon^{-1}$.

\section{REPLICA DEPENDENT COUPLING}

In this section we present some numerical results in the case in which the system-bath coupling depends on the qubit replica. If the coupling functions $\left\{g_{k}(i)\right\}$ and/or the qubit energies $\left\{\epsilon_{i}\right\}$ depend on the qubit replica, the total spin operator $S^{2}$ is no longer a constant of the motion. This situation is expected to be more realistic than the one previously assumed in that the latter amounts to have a bath with an infinite (i.e. very large) coherence length. In this case the decomposition (13) is not invariant: the dynamics results in a non-trivial mixing of the $s u(2)$-irreducible sectors $\mathcal{H}_{I}(S, r)$. In particular one has $S^{-} \mathcal{H}_{1}^{A} \neq 0$, therefore the vectors $\left|\psi^{a}\right\rangle$ can decay. In other words the loss of the symmetry constraint allows the dissipation-decoherence induced by the bath to invade the whole Hilbert space. We choose $g_{k}(i)=g_{0} \cos (k i / \xi)$, where $\xi$ is a parameter related to the bath coherence length (so that for $\xi=\infty$ we recover the results of the previous sections). In figure (位) are reported the plots of $F(t)$ with initial condition in $\mathcal{H}_{1}^{A}$ for different $\xi$ 's. 


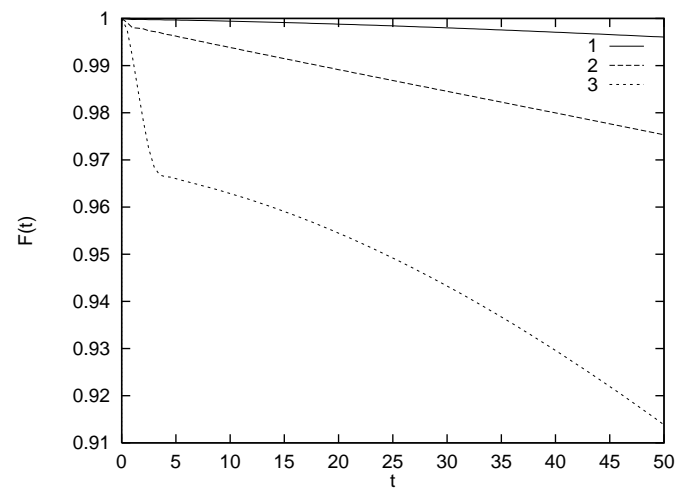

FIG. 4. Fidelity as a function of time for $\left|\psi_{0}^{s}\right\rangle \in \mathcal{H}_{1}^{A}$, with $\xi=10(1), \xi=5(2), \xi=1(3)$. The coupling function is $g_{k}(i)=g_{0} \cos (k i / \xi) . N=2, N_{b}=200, g_{0}=0.01$. The time unit is $\epsilon^{-1}$.

Figure (5) shows the behaviour of $F(t)$ for small times with $\xi=1$. Notably one observes that the initial condition $\left|\psi_{0}^{s}\right\rangle \in \mathcal{H}_{1}^{A}$, exhibits a faster fidelity decay with respect to $\left|\psi_{0}^{s}\right\rangle \in \mathcal{H}_{1}^{s y m}$, for short times $t<t_{c}$. For longer times, with obvious meaning of the notation, $F_{A}(t)>F_{\text {sym }}(t)$. The numerical simulations show in any case that $\bar{F}_{A}>\bar{F}_{\text {sym }}$, the bar denoting temporal average.

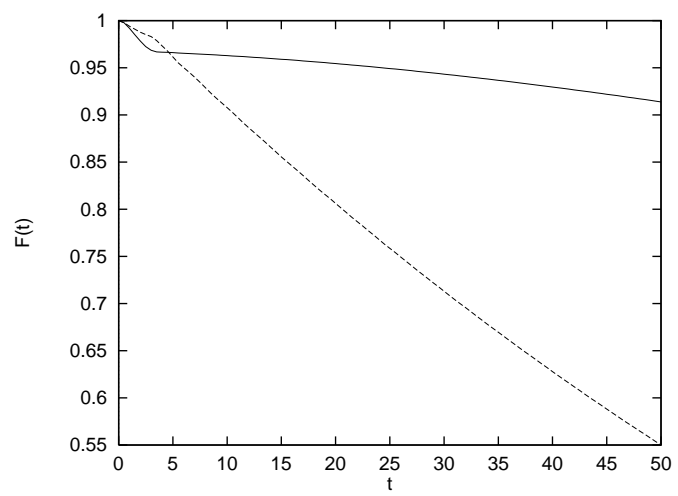

FIG. 5. Fidelity as function of time for $\left|\psi_{0}\right\rangle=\left|\psi^{\text {sym }}\right\rangle$, (dashed line) and $\left|\psi_{0}^{s}\right\rangle \in \mathcal{H}_{1}^{A}$, (solid line). The coupling function is $g_{k}(i)=g_{0} \cos (k i) . N=2, N_{b}=200, g_{0}=0.01$. The time unit is $\epsilon^{-1}$.

\section{CONCLUSIONS}

In this paper we have presented the study of a physical model for a quantum register coupled with the environment. Information is encoded in the quantum state of the register. The register consists of $N$ non-interacting replicas of a two-level system (i.e. a $N$-qubits register). The register environment is described by a bosonic bath consisting of $N_{b}$ modes, with $N_{b} \gg N$. Its coupling with the register is realized by the exchange of elementary quanta of energy. The resulting dynamics of the register is dissipative; in the weak coupling regime, energy and information are irreversibly lost into the bath. Even though the model is non-trivial, exact analytical as well as numerical results can be obtained thanks to the existence of a constant of the motion (excitation number) that leads to a decomposition of the total Hilbert space in dynamically independent sectors. Assuming that the environment couples in the same way with all the register qubits one has a further splitting of the sectors according to the irreducible representations of the spin $s u(2)$ algebra. Each $s u(2)$ lowest vector is decoupled from the bath vacuum fluctuations and therefore, at zero temperature, is decoherence free. The smallest subspace in which one can have non-trivial physics is the one-excitation sector $\mathcal{H}_{1}$. The dimension of $\mathcal{H}_{1}$ scales linearly in the total number of degrees of freedom, therefore a thorough analysis of the dynamics in $\mathcal{H}_{1}$, can be performed by means of exact numerical diagonalization of the model Hamiltonian. The temporal dependence of quantity of interest, such as fidelity and entropy have been studied. The asymptotic behaviour depends on the symmetry content of the initial state. Smaller is the projection of the initial state over the vacuum $s u(2)$-sector $\mathcal{H}^{s y m}$, greater is the fidelity. In particular a complete energy-information loss occurs only when the state belongs to $\mathcal{H}^{\text {sym }}$. Some numerical results for bath-system coupling dependent on the qubit are also presented. In this more realistic situation the $s u(2)$-structure is unstable: dissipation and decoherence affects the whole Hilbert space and then safe encondings no longer exists. Nevertheless our results shows that, on long time scales, the average fidelity of the previously noiseless states is still greater of the one of the other states. This suggest that the symmetry-based protection of quantum state suggested in [4] can be valuable in the general case. This last issue, along with the necessary finite temperature generalizations, worth further investigations.

\section{ACKNOWLEDGMENTS}

Stimulating discussions with M. Rasetti and R. Zecchina are gratefully aknowledged. The author also thanks C. Calandra and G. Santoro for providing him access to the CICAIA of the Modena University, and Elsag-Bailey for financial support 
[1] For reviews, see D.P. DiVincenzo, Science 270, 255 (1995); A. Ekert and R. Josza, Revs. Mod. Phys. 68, 733, (1996)

[2] For a review see W.H. Zurek Physics Today 44, 36 (1991); W. G. Unruh, Phys. Rev. A 51, 992 (1992) P.W. Shor, W. H. Zurek, I.L. Chuang, and R. Laflamme, Science 270, 1633 (1995).

[3] P.W. Shor, Phys. Rev. A 52, 2493 (1995); C.H. Bennet, D.P. DiVincenzo, J. A. Smolin, and W.K. Wootters, Phys. Rev. A A, 3284 (1996); R. Laflamme, C. Miquel,J.P. Paz, and W..H. Zurek, Phys. Rev. Lett. bf 76, 198 (1996); A. M. Steane, Phys. Rev. Lett. 77, 793 (1996); A. Ekert, C. Macchiavello, Phys. Rev. Lett. 77, 2585 (1996);

[4] P. Zanardi, M. Rasetti Noiseless Quantum Codes, submitted to Phys. Rev. Lett, December 1996
[5] Dynamical Groups and Spectrum Generating Algebras, A. Bohm, Y. Néeman, and A.O. Barut, eds.; World Scientific, Singapore, 1988

[6] A. Garg, Phys. Rev. Lett.. 77, 964 (1996)

[7] E. B. Davies Quantum theory of open systems, Academic Press, London (1976)

[8] K. Hepp, E. Lieb, Phys. Rev. A 8, 2517 (1973)

[9] E.T. Jaynes, F. W. Cummings, Proc. IEEE 51, 89 (1963)

[10] A.J. Legget, S. Chakravarty, A.T. Dorsey, M. P. A. Fischer, A. Garg, W. Zwerger, Rev. Mod. Phys. 59, 1 (1987)

[11] R. Davidson, J.J. Kozak, J. Math. Phys. 8, 903 (1971); J. Math. Phys. 14, 414 (1973)

[12] B. Schumacher, Phys. Rev. A, 51, 2738 (1995)

[13] Eberly, Norozhny, Sanchez-Mondragon, Phys. Rev. Lett. 44, 236 (1981) 